\title{
The Stability and Design Optimization of Qiyun Pit Slope Based on Reliability Theory
}

\author{
Ding Jihui ${ }^{1 \mathrm{a}}$, Xiang Mengjia ${ }^{1 \mathrm{~b}}$, Shen $\mathrm{Yu}^{2 \mathrm{c}}$, Wei Yaxing ${ }^{1 \mathrm{~d}}$, Huang Jie ${ }^{1 \mathrm{e}}$ \\ ${ }^{1}$ Department of Civil Engineering, College of Civil Engineering and Architecture, \\ Hebei University, Baoding, 071002, China \\ ${ }^{2}$ Supervision office, Shanghai Electronics Industry School, Minhang District, Shanghai 200240, China \\ Email: a dingjihui@126.com; b xmj517@foxmail.com; c Shenyu6@163.com; d 553629381@qq.com; \\ e 841321413@qq.com
}

\begin{abstract}
Taking a rock slope in Qinhuangdao as an example, the non-probabilistic reliability model of rock slope stability is established, and the interval method is applied to the non-probabilistic reliability analysis of rock slope stability. It can provide a simple and effective calculation method for the stability analysis of actual slope engineering. According to the safety factor and reliability index, the slope angle is optimized, continuously decreasing 1 degree until the safety factor F is greater than 1.2 on the basis of the original angle. The influence of rock mass parameter variability on the non-probabilistic reliability index of rock slope stability is discussed. The influence of rock mass parameter change on the position of sliding surface is considered. According to the position of different sliding surfaces, the relationship between the variability of rock weight $(\gamma)$, cohesion $(c)$, internal friction angle $(\varphi)$ and non-probabilistic reliability index $(\eta)$ is analyzed. Variations of the geotechnical parameters on slope stability are mostly negative. Therefore, in the actual project, the range of the geometric parameters must be strictly controlled.
\end{abstract}

Keywords: Interval analysis; non-probabilistic reliability; slope angle optimization; sliding surface; sensitivity analysis

\section{Introduction}

The safety and stability of rock slope has great influence on engineering, and it is very important to evaluate the stability of rock slope quickly and accurately[1]. At present, the deterministic analysis method based on rigid body limit equilibrium analysis is usually used in slope stability analysis of geotechnical engineering, and the safety factor is used as the slope stability evaluation index[2]. The method is simple and practical, however, because the influence of rock and soil slope stability factors is complex and the values of rock mass parameters are interval, the safety factor cannot be determined considering any inherent variability in the design parameters, so the analysis result is difficult to reflect the engineering practice[3-4]. Therefore, it is more appropriate to use reliability theory and method to study and solve the actual slope stability problem. It is more suitable to use the possible range instead of a simple number.

The reliability of slope is the traditional theory of probability theory and mathematical statistics applied in slope engineering results, the reliability method is to use probability theory and fuzzy theory to deal with the uncertainty, but the solution often requires a large amount of data to determine the parameters of probability distribution and membership function, and with the lack of sufficient data, the calculation results of the probabilistic model there may have a large deviation[1,5]. The probability reliability is very sensitive to the probability model parameters, and the small error of probability data may lead to larger errors in the reliability calculation results[6]. To solve the above problems, based on the comprehensive analysis of the interval of geotechnical parameters, the non-probabilistic reliability model of slope stability is established by introducing interval analysis method and non-probability reliability theory, and the non-probabilistic reliability analysis of slope is carried out. It can provide a simple and effective calculation method for the stability analysis of actual slope engineering. 


\section{Brief Introduction to Non-Probability Reliability Interval Analysis Method}

\subsection{Interval Method}

Interval mathematics is the theoretical foundation of interval non-probabilistic reliability research[7]. Suppose that in the real field $\mathrm{R}$, there is the following relationship for any given two real numbers $\underline{x}$ and $\bar{x}: \underline{x}, \bar{x} \in R, \underline{x} \leq \bar{x}$, then the bounded closed interval in the real field can be expressed as:

$$
X=[\underline{x}, \bar{x}]=\{x \in R \mid \underline{x} \leq x \leq \bar{x}\}
$$

where, $\mathrm{x}$ is interval variable;

$\mathrm{X}$ is the interval of interval variable $\mathrm{x}$;

$\underline{x}, \bar{x}$ are the upper and lower endpoints of the interval.

For any interval $X=[\underline{x}, \bar{x}]$, its interval mean is $x^{c}=(\underline{x}+\bar{x}) / 2$, interval radius is $x^{r}=(\bar{x}-\underline{x}) / 2$, interval width is $x^{I}=2 x^{r}=\bar{x}-\underline{x}$, then the interval $\mathrm{X}$ can be expressed as:

$$
X=[\underline{x}, \bar{x}]=\left[x^{c}-x^{r}, x^{c}+x^{r}\right]
$$

\subsection{Non-Probabilistic Reliability Principle}

The reliability of slope is affected by many factors, such as rock weight $(\gamma)$, cohesion $(c)$, internal friction angle $(\varphi)$, elastic modulus $(E)$, Poisson ratio $(v)$, but also has a range of these variables, the performance function was determined by the slope instability criterion:

$$
M=g(x)=g\left(x_{1}, x_{2}, \cdots, x_{i}\right)=R-S
$$

where, $x_{i}$ are rock mass parameter variables;

$\mathrm{R}$ is anti sliding force;

$\mathrm{S}$ is Sliding force.

If the function $g(x)$ is the continuous function of each interval variable $x_{i}$; then the corresponding quantity $\mathrm{M}$ is an interval variable.

Then the interval non-probabilistic reliability index $\eta$ can be defined as follows:

$$
\eta=\frac{M^{c}}{M^{r}}=\frac{\bar{M}+\underline{M}}{\bar{M}-\underline{M}}
$$

where, $M^{c}$ is the mean value of interval variable $\mathrm{M}$;

$M^{r}$ is the deviation of interval variable $\mathrm{M}$.

According to the reliability theory, there are three working states of slope, that is, safety state, limit state and failure state. The limit equation of state $g(x)=0$ divides the parameter variables of the slope into two parts, the failure domain $g(x)<0$ and the safety domain $g(x)>0$, which represent the failure state and the safety state of the slope respectively.

(1) When the non-probabilistic reliability index $\eta>1$, for any variable parameter for the slope $x_{i} \in X(i=1,2, \cdots, n)$, there is the limit state equation $g(x)>0$ of slope, indicating the slope is in a safe state.

(2) When $\eta<-1$, for any $x_{i} \in X(i=1,2, \cdots, n)$, there is $g(x)<0$, and indicating the slope is in failure state.

(3) When $-1<\eta<1$, for any $x_{i} \in X(i=1,2, \cdots, n), g(x)>0$ and $g(x)<0$ are possible, and the slope is in the state of failure or safety. However, when all the uncertain parameters of the slope take the number of intervals, it can be considered that the slope may only be in two states, namely, the safety state and the failure state. Therefore, from the interval meaning and the actual situation of the project, the slope under third kinds of situations is not reliable.

From the expression of $(\eta)$, the larger the dimensionless $(\eta)$ value is, the farther the slope fluctuation 
range is from the failure surface, that is to say, the higher the slope safety is [8]. Therefore, $(\eta)$ can be used as a measure of slope stability reliability.

\section{$3 \quad$ Engineering Example}

\subsection{Basic Parameters}

Taking a slope of Qinhuangdao city in Hebei Province as an example, the lithology of the slope is mainly granite, with joint development, slope height is $41 \mathrm{~m}$, and slope angle is $46^{\circ}$. The experience shows that the calculation accuracy is ideal when the slope boundary is 2 times higher than the slope height [9]. Therefore, the calculated area is 2 times higher in horizontal extension of slope height, and the vertical extension is 2 times higher of slope height. The calculation model is shown in Figure 1, unit m. The first layer of the rock slope is broken middle weathered granite, and the second layer is moderately weathered granite. According to the survey data and relevant specifications, the values of rock slope parameters are shown in table 1.

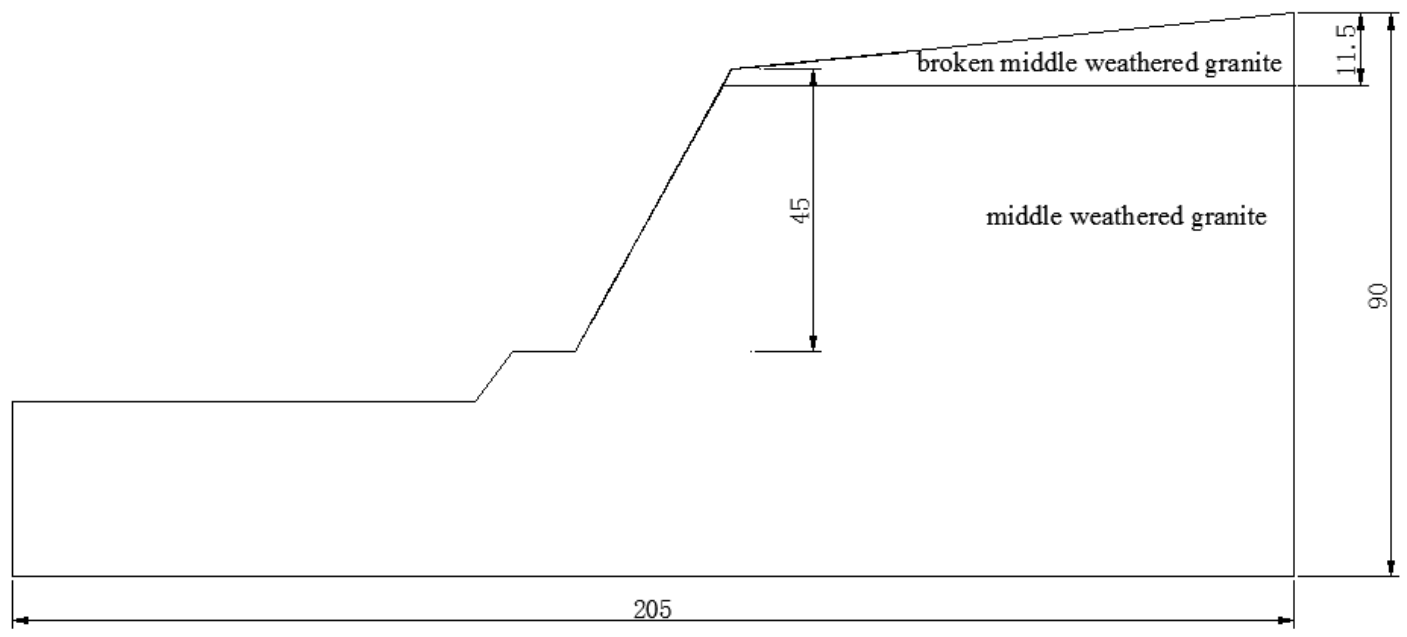

Figure 1. Dimension of slope calculation model

Table 1. Values of rock slope parameters

\begin{tabular}{ccccc}
\hline Rock type & Cohesive force $/ \mathrm{Pa}$ & $\mathrm{E} / \mathrm{Pa}$ & Poisson ratio & Internal friction angle $/^{\circ}$ \\
\hline $\begin{array}{c}\text { Broken middle weathered } \\
\text { granite }\end{array}$ & 117500 & $1.00 \mathrm{E}+10$ & 0.36 & 25 \\
middle weathered granite & 142500 & $3.00 \mathrm{E}+10$ & 0.35 & 27 \\
\hline
\end{tabular}

\subsection{Safety Factor}

For the rock slope, because the actual rock mass contains a lot of discontinuities such as different structures, occurrences and characteristics (such as the level, joints, cracks, weak interlayer, rock veins and fault zone, etc.), it brings great difficulties to the stability analysis of rock slope, so it is difficult to determine the location of the sliding surface when the slope is unstable [10]. Using the finite element software ANSYS to carry out the numerical simulation of the slope, in order to determine the slope stability when the position of the sliding surface, the full reduction safety coefficient was 1.17 , the local reduction safety coefficient was 1.174, using the Swedish slice method to calculate $\mathrm{F}=1.06$, using simplified Bishop to calculate $\mathrm{F}=1.13$.

\subsection{Function of Slope Reliability Analysis}

Based on the Swedish slice method, the function of slope reliability analysis is obtained: 


$$
M=R-S=\tan \varphi \sum_{i=1}^{n} W_{i} \cos \alpha_{i}+c \hat{L}-\sum_{i=1}^{n} W_{i} \sin \alpha_{i}
$$

where, $W_{i}$ is gravity of i soil strip, $W_{i}=\gamma b_{i} h_{i}$;

$\alpha_{i}$ is the angle between the normal (radius) of the sliding surface and vertical line of the i slip strip $\left(^{\circ}\right.$ );

$l_{i}$ is arc length of sliding surface of i soil strip, m;

$b$ is width of i soil strip, m;

$h$ is center height of i soil strip, m;

$\varphi_{i}$ is internal friction angle on sliding surface, $\left(^{\circ}\right)$;

$c_{i}$ is cohesive force of soil on sliding surface, $\mathrm{kPa}$.

According to the relevant specifications and combined with the actual situation of slope, the variation coefficient of each parameter is respectively: $\delta_{\gamma}=0.05, \delta_{c}=0.15, \delta_{\varphi}=0.1$. The slope reliability index $\eta$ $=0.981$ can be obtained by introducing the parameters of the slope into (5) and then according to formula (4), which indicates that the slope is unstable and needs to be optimized.

Based on the simplified Bishop method, the slope reliability analysis function:

$$
\begin{gathered}
M=R-S=\sum_{i=1}^{n} \frac{1}{m_{\alpha i}}\left[W_{i} \tan \varphi_{i}+c_{i} l_{i} \cos \alpha_{i}\right]-\sum_{i=1}^{n} W_{i} \sin \alpha_{i} \\
m_{\alpha i}=\cos \alpha_{i}+\frac{1}{F} \tan \varphi_{i} \sin \alpha_{i}
\end{gathered}
$$

In formula (6) and formula (7), the meaning of each parameter is the same as that of formula (5).

The slope reliability index $\eta=1.393$ can be obtained by introducing $\delta_{\gamma}=0.05, \delta_{c}=0.15, \delta_{\varphi}=0.1$ into (5) and then according to formula (4), which indicates that the slope is stable.

\subsection{Optimal Design}

Practice shows that the slope safety factor greater than 1 may also bring damage, so the slope safety factor $\mathrm{F}$ should be greater than 1.2. On the basis of the size of the original slope, the slope angle is $61^{\circ}$, and the slope angle is decreased by $1^{\circ}$. After each update, the safety factor $\mathrm{F}$ of the new slope is recalculated until the safety factor $\mathrm{F}$ is greater than $1.2[11]$.

In the optimization process, the relationship curves between the slope safety factor and the non-probabilistic reliability and the slope angle are shown in figures 2 and 3 . We can see from Figure 2 , local reduction calculation of safety factor is slightly greater than full reduction calculation of safety factor. The Swedish strip method will be smaller than the actual situation, $10 \% \sim 20 \%$, partial to safety. The results of the Bishop's method are closer to the actual situation. As can be seen from Fig. 3, according to the Swedish method, the reliability index is increased from 0.981 to 1.554 , from unstable to stable. The reliability index calculated from the simplified Bishop method is increased from 1.393 to 2.02, indicating that the slope is more stable after cutting. The slope is reduced by 5 degrees on the basis of the original slope angle of $61^{\circ}$, the slope safety factor is greater than 1.2 , and the reliability is greater than 1 , which indicates that the slope meets the safety requirements.

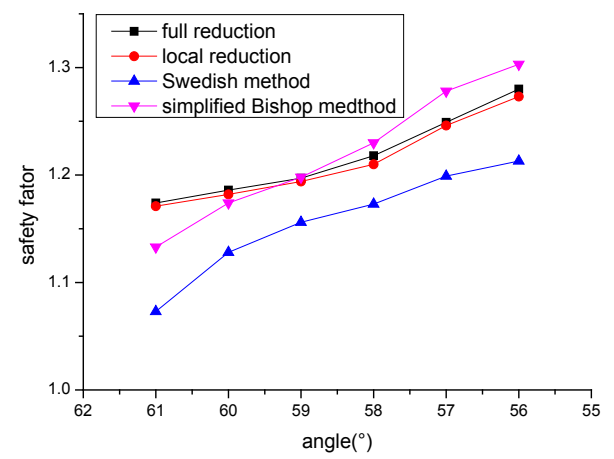

Figure 2. Relationship between safety and slope angle 


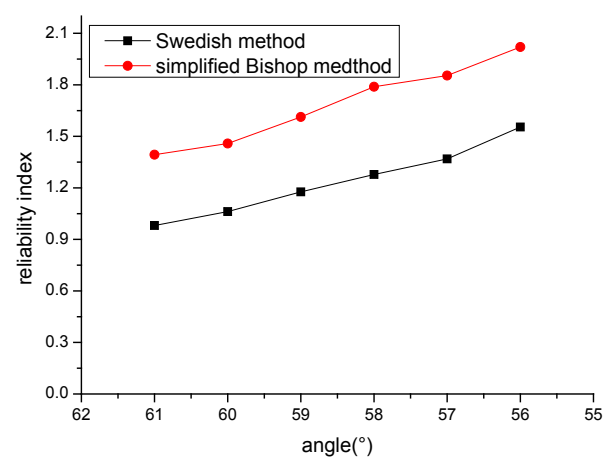

Figure 3. Relationship between reliability factor index and slope angle

\section{Sensitivity Analysis of Rock Parameters}

In order to further explore the influence of rock mass parameter variability on the non-portability reliability index of rock slope stability, combined with the above engineering example, discussed the influence of variation of rock weight $(\gamma)$, cohesion (c), internal friction angle $(\varphi)$ on slope stability of the non-probabilistic reliability index $(\eta)$. When the sensitivity of the parameters is analyzed, it is assumed that the values of other parameters are unchanged.

\subsection{Influence of Variability of Rock Index on Slipping Surface}

When $\delta_{c}=0.15, \delta_{\varphi}=0.1$, only change the interval of the rock weight $(\gamma)$, numerical simulation shows that the position and shape of the sliding surface are basically unchanged.

When $\delta_{\gamma}=0.05, \delta_{\varphi}=0.1$, only change the interval of cohesion (c), numerical simulation of sliding surface position as shown in Figure 4, the position of the sliding surface of the slope gradually moves from the inside of the slope to the near slope surface. The distance between slope top slippery point and slope shoulder is gradually decreased.

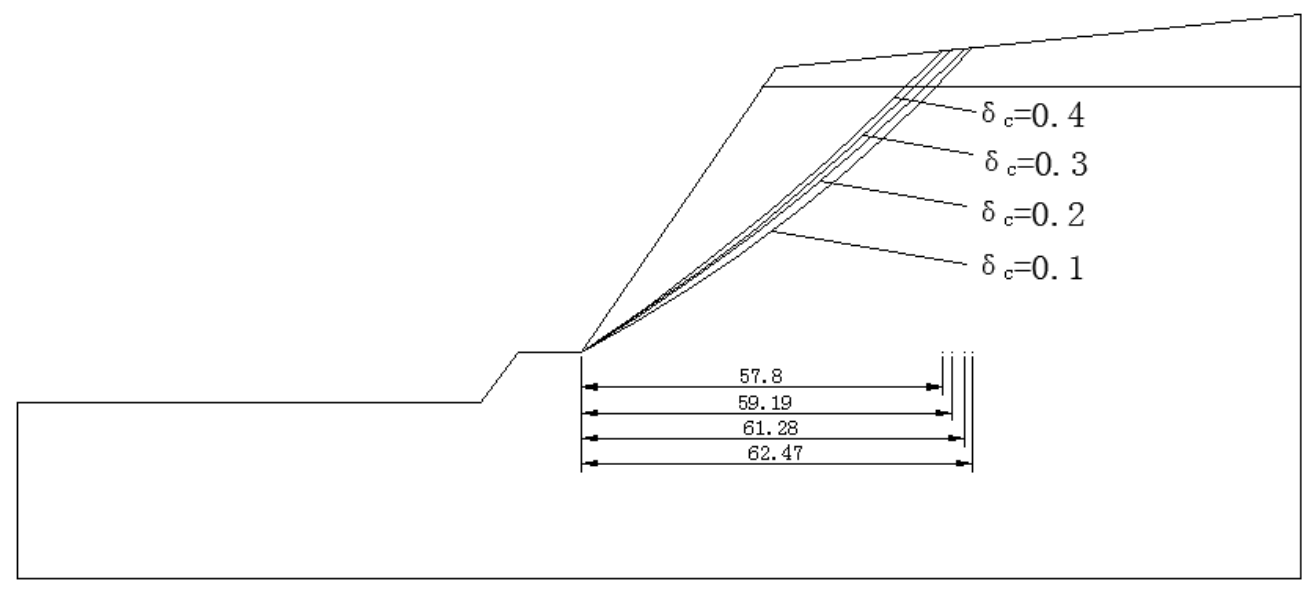

Figure 4. Influence of cohesive force $\mathrm{C}$ change on slipping surface of slope

When $\delta_{\gamma}=0.05, \delta_{c}=0.15$, only change the interval of friction angle $(\varphi)$, numerical simulation of sliding surface position as shown in Figure 5 , the position of the sliding surface of the slope gradually moves from the near slope surface to the inside of the slope. The distance between slope top slippery point and slope shoulder is gradually increased. 


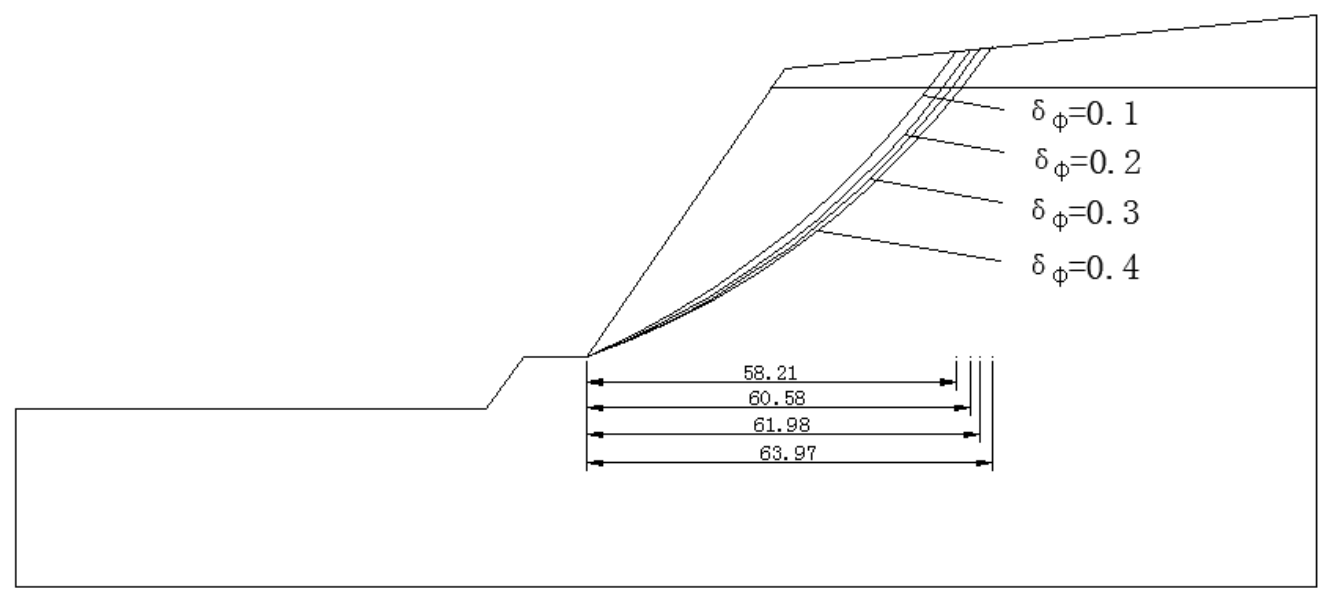

Figure 5. Influence of change of internal friction angle on sliding surface of slope

\subsection{Influence of Variability Rock Index on Reliability Index $(\eta)$}

The relationship between the variation coefficient rock mass and non-probabilistic reliability index $(\eta)$ is shown in figure 6 . It can be seen from figure 6 :

(1) With the variation coefficient rock weight $(\gamma)$ increased from 0.05 to 0.3 , the non-probabilistic reliability index decreased from 1.678 to 0.937 by the Swedish slice method, the reliability index $(\eta)$ fell $44.16 \%$ points, non-probabilistic reliability index through the Simplified Bishop method decreased from 2.020 to 1.326 , the reliability index $(\eta)$ fell $34.36 \%$.

(2) With the variation coefficient cohesion (c) increased from 0.1 to 0.4 , the non-probabilistic reliability index decreased from 2.162 to 0.765 by the Swedish slice method, the reliability index $(\eta)$ decreased by $64.62 \%$, non-probabilistic reliability index through the Simplified Bishop method decreased from 2.052 to 1.019, the reliability index $(\eta)$ fell $50.34 \%$.

(3) With the variation coefficient internal friction angle $(\varphi)$ from 0.1 to 0.4 , the non-probabilistic reliability index decreased from 1.678 to 0.721 by the Swedish slice method, the reliability index decreased by $57.03 \%$, non-probabilistic reliability index $(\eta)$ through the Simplified Bishop method decreased from 2.02 to 0.9 , the reliability index $(\eta)$ fell $55.45 \%$.

It is shown that the influence of variation coefficient rock weight $(\gamma)$, cohesion $(\mathrm{c})$ and internal friction angle $(\varphi)$ on slope stability non-probability reliability index $(\eta)$ is very significant. The greater the variation coefficient, the more detrimental to the stability of the slope, and showed a nonlinear relationship. Among them, the variation coefficient cohesion $\mathrm{c}$ and internal friction angle have significant influences on the non-probabilistic reliability index $(\eta)$ of slope stability.
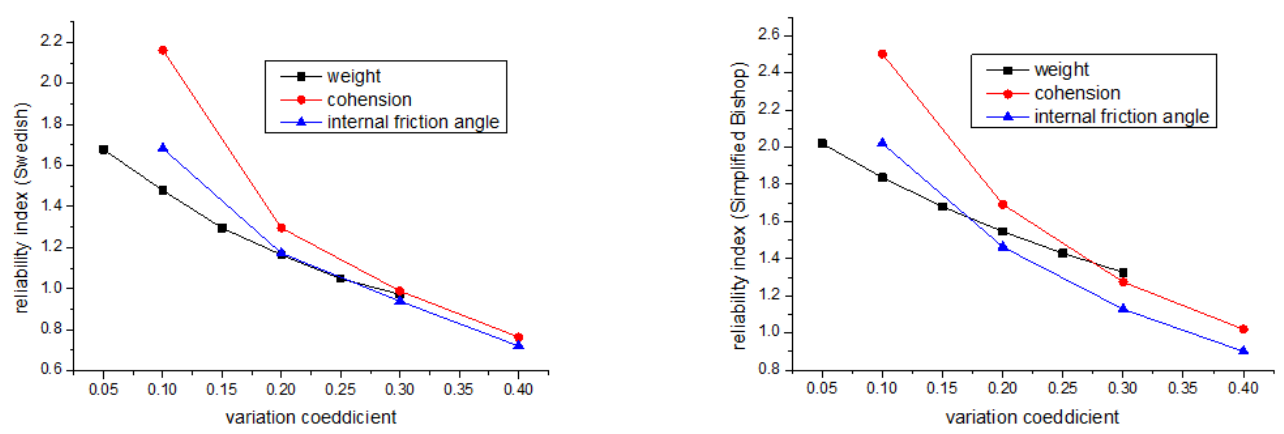

Figure 6. Relationship curves between variation coefficient rock parameters and reliability index $(\eta)$ 


\section{Conclusion}

Analyzing the main geotechnical parameters affecting slope stability based on the traditional limit equilibrium method, and applied the interval method to the non-probabilistic reliability analysis of slope stability. In the absence of a large number of sample parameters, quantifying the slope stability analysis results, and obtaining the non-probability reliability calculation results of slope.

(1) The safety factor is calculated by different methods, showing that the Swedish strip method will be smaller than other calculation methods by $10 \% \sim 20 \%$, partial to safety. The results of the Bishop's method are closer to the actual situation. Local reduction calculation of safety factor is slightly greater than full reduction calculation of safety factor by the numerical simulation [11].

(2) The non-probabilistic reliability index overcomes the shortcoming of the large sample test data required by the probability reliability index, and carries out effective safety evaluation for the slope engineering, which provides a simple and effective calculation method for the stability analysis of the actual slope engineering.

(3) The results of parameter sensitivity analysis show variations of the geotechnical parameters on slope stability are mostly negative. The accurate geotechnical parameters are of great significance to the slope stability evaluation. Therefore, in the actual project, the range of the geometric parameters must be strictly controlled [6].

\section{References}

1. Han Xiaofeng, Chen Haijun. "Non-probabilistic reliability analysis based on the ellipsoidal model of rock slope stability," Journal of Xihua University(Natural Science), 2015, 34(6):96-100.

2. Chen Guoqing, Huang Runqiu, Shi Yuchuan, et al. "Stability analysis of slope based on dynamic and whole strength reduction methods," Chinese Journal of Rock Mechanics and Engineering, 2014, 33(2):243-256.

3. Cao Wengui, Zhang Yongjie. "Non-probabilistic fuzzy reliability analysis of slope stability based on interval interconnection method," China Civil Engineering Journal, 2007, 40(11):64-69.

4. Ma Chunhui, Yang Jie, Chen Lin, et al. "Slope stability analysis based on HS-RVM with mixed kernel," Chinese Journal of Rock Mechanics and Engineering, 2017(a01):3409-3415.

5. Johari A, Javadi A A. "Reliability assessment of infinite slope stability using the jointly distributed random variables method," Scientia Iranica, 2012, 19(3):423-429.

6. Yu Shengfei, Chen Zhengyu, Zhang Mingrui, et al. "Interval analysis model of geomaterial parameters for uncertainties in slope stability assessment," Journal of Engineering Geology, 2012, 20(2):228-233.

7. Kang Jianchao. "Non-probabilistic Reliability Studies of Tunnel Lining Systems Based on Interval Theory," Shijiangzhuang Tiedao University, 2016.

8. Tu Yiqiang, Wang Jingquan, Jiang Kebin. "Non-probabilistic Reliability Analysis of Structural System Based on Interval Analysis," Journal of PLA University of Science and Technology: Natural Science Edition, 2003, 4(2):48-51

9. Lai Yongbiao, Hu Renxi, Huang Shuzhen. "ANSYS 11.0 typical example of finite element analysis of civil engineering," Electronic Industry Press, 2007.

10. Jin Fucheng. "Research on failure criterias and critical slip surfaces of rockmass slope," Hehai University, 2007

11. Yu Shengfei. "The research of stability analysis and non-probabilistic reliability evaluation of slopes based on interval uncertainty medthod," Nanjing University, 2012. 